\title{
Tachyon Condensation in Large Magnetic Fields with Background Independent String Field Theory
}

\author{
Lorenzo Cornalba \\ École Normale Supérieure \\ Paris, France \\ cornalba@lpt.ens.fr
}

October 2000

\begin{abstract}
We discuss the problem of tachyon condensation in the framework of background independent open string field theory. We show, in particular, that the computation of the string field theory action simplifies considerably if one looks at closed string backgrounds with a large $B_{a b}$ field, and can be carried out exactly for a generic tachyon profile. We confirm previous results on the form of the exact tachyon potential, and we find, within this framework, solitonic solutions which correspond to lower dimensional unstable branes.
\end{abstract}




\section{Introduction}

The problem of tachyon condensation on unstable branes has received lately a lot of attention [22, 3, 4, 5, 7, 8, 9, 21, 11, 12, 13, 14, 15, 23, 24, 25, and considerable progress has been made following the initial conjectures of Sen (see [14, 15] and references therein). The problem is most easily studied in the context of bosonic open string physics, where the relative simplicity of the theory has led both to numerical as well as exacts checks of the conjecture.

Questions on tachyon condensation are most naturally addressed within string field theory [10, 17], since we are interested in processes which connect two distinct vacua of the theory, and which are by their very nature off-shell non-perturbative phenomena. More precisely, Sen's conjecture states that we should find a variety of stationary points of the string field theory action, corresponding to branes of various dimensions. Moreover, the value of the action at these stationary points should equal the energy of the corresponding brane configurations. Finally the minimum of the string field theory action should be given by a unique transitionally invariant vacuum which contains no open strings at all.

¿From a practical point of view, two are the questions that need to be answered. First of all, one needs to compute the tachyon potential, and show that the difference in energy between the maximum and the minimum corresponds to the energy of a single 25-brane. Second, on needs to find, within the string field theory, classical solitons which correspond to branes of lower dimensions.

These questions have been mostly addressed within the framework of Witten's cubic Chern-Simons string field theory [10], and have been studied in the two different closed string backgrounds, either with vanishing NSNS two-form field $B_{a b}$, or with an infinitely large $B_{a b}$. In the first case, most results are numerical [2, 5, 3, 4], and have led, using the level truncation scheme, to computations of the tachyon potential which confirm Sen's conjecture to approximately 1\%. Tensions of branes are more difficult to obtain in this case, since the corresponding solitons are expected to have a characteristic size of order $l_{s}$, and therefore all derivative corrections to the string action will then contribute with the same weight. Indeed, solitons can be found even restricting the action to two derivatives [7, 8, 9], but the energies obtained are usually only qualitatively correct. Still in the $B_{a b}=0$ regime, an analytical construction of the stable vacuum has been given in [6]. In the limit of large $B_{a b}$ nothing new can be said about the tachyon potential, but solitons corresponding to the various branes can be explicitly characterized and energies can be computed exactly [25, 23]. This is the case since the presence of a magnetic field introduces a new length scale which becomes much larger then the effective string scale, and 
which controls the size of the solitonic solutions. Therefore $\alpha^{\prime}$ derivative corrections to the action become irrelevant in the computation of the energies. The unique information which is needed in order to compute tensions exactly is the value of the tachyon potential at the extremum points, which is assumed from the conjecture of Sen.

A different approach to the problems discussed above has been proposed in [21, 22], and is based on an alternative background independent formulation of open string field theory, which was originally proposed in [16] and then subsequently developed in [17, 18, 19, 20]. The basic data is a two dimensional field theory defined on a disk string world-sheet $\Sigma$ (the disk being equipped, for definiteness, with the standard metric). The action is conformal in the interior of $\Sigma$, and is the one defining the closed string background. On the boundary, on the other hand, one can generically insert any operator (of ghost number zero) built from the fields of the theory and their derivatives. Every single boundary operator will come with its own coupling constant $g$ and we should consider the space of all coupling constants as the configuration space of string field theory. The action functional $S(g)$ is then very simply computed starting from the partition function $Z(g)$ of the above theory, as well as from the $\beta$-function RG flow in the configuration space of coupling constants $g$.

Already in the early work of [16] it was conjectured that a stable vacuum could correspond to a breaking of Lorentz invariance from $S O(1,25)$ to $S O(2) \times S O(1,23)$, or, in modern language, to a decay from a 25 to a 23 -brane. More recently, in [21, the authors compute, for generic tachyon profile and for vanishing $B_{a b}$, the effective action up to two derivatives. In particular, they are able to compute exactly the tachyon potential, precisely confirming Sen's conjecture. Moreover, in [22] the authors have shown that, if one works with the same closed string background with $B_{a b}=0$, and if one considers as a boundary operator a tachyon profile which is quadratic in the space-time coordinates, then one can compute the action to all orders in derivatives (based on [18]). Moreover one can describe explicitly the solitons corresponding to the lower dimensional branes, and compute exactly their energy.

In this letter we show that, if one considers the procedure of [22, 21] but concentrates, as a closed string background, on the limit of large $B_{a b}$, then one can compute the action for generic tachyon profiles extremely easily. We recover the expected form of the potential, and we compute energies of solitonic solutions. All results agree with the known expectations, and we make some contact with the usual formulation [25, 24] of the problem in cubic open string field theory in the large $B_{a b}$ limit. 


\section{$2 \quad$ Large Magnetic Field Limit}

We study a bosonic open string moving in Euclidean space-time, and we take the metric to be, for simplicity, equal to $\delta_{a b}$. We will concentrate, in particular, on the motion in two of the Euclidean directions, with coordinates $x^{1}$ and $x^{2}$, and we shall collectively call the other 24 perpendicular coordinates $y_{\perp}$. We will finally have, as a background field, a constant NSNS 2-form potential in the 1-2 direction given by

$$
B_{12}=B
$$

Units will be such that $2 \pi \alpha^{\prime}=1$.

We consider, following [17, 19, 20], string actions defined on the unit disk $\Sigma \subset \mathbb{C}$ (endowed with the standard flat metric) of the general form

$$
I=I_{0}+\frac{1}{2 \pi} \int_{\partial \Sigma} d \tau V
$$

In the above equation, the action $I_{0}$ is given by

$$
\begin{aligned}
I_{0}= & \frac{1}{2} \delta_{a b} \int_{\Sigma} d X^{a} \wedge \star d X^{a}+i B \int_{\Sigma} d X^{1} \wedge d X^{2} \\
& + \text { terms in } Y_{\perp} \text { and ghost fields, }
\end{aligned}
$$

and is the usual conformal action defined on the interior of the disk $\Sigma$ which describes the motion of the string in the chosen closed string background. On the other hand, the operator $V$ inserted on the boundary is a general linear combination

$$
V=V_{i} g^{i}
$$

where the operators $V_{i}$ span the set of all possible boundary operators built only from the matter part of the theory (the most general construction, which does not assume a decoupling of matter and ghosts, is given in [17], but we will not need it in the sequel).

In the rest of this letter, we will actually restrict our attention, as indicated at the beginning of the section, to boundary operators $V_{i}$ built only from the matter fields $X^{a}$ and independent of the transverse $Y_{\perp}$. This means that we are considering physical configurations which are invariant under translations in the transverse directions $y_{\perp}$.

The main result of [20] is that, if one considers the partition function

$$
Z(g)=\int D X e^{-I(X)}
$$


then the classical background independent effective action which describes open string boundary interactions is given by

$$
S=\left(1+\beta^{i} \frac{\partial}{\partial g^{i}}\right) Z,
$$

where the functions $\beta^{i}=d g^{i} / d \ln M$ are the usual $\beta$-functions ( $M$ is a mass scale) associated to the couplings $g^{i}$. To first order in the couplings, the $\beta$-functions are given by

$$
\beta^{i}=\left(\Delta_{i}-1\right) g^{i}+o\left(g^{2}\right)
$$

where $\Delta_{i}$ is the dimension of the operator $V_{i}$.

We will use the above general prescription to compute the tachyon field effective action in the limit of large $B \rightarrow \infty$. Before doing so, let us though discuss some issues regarding the normalization (see also [11]) of the path integral (11), by considering the action $S(0)$ at zero value of the coupling constants $g^{i}$. On one hand, it is well known that the value of the action for a brane (maximal 25-brane, since we are considering open strings with Neumann boundary conditions) with a constant magnetic field $B_{12}=B$ is given by the Born-Infeld value

$$
S(0)=T_{25} V_{\perp} \int d^{2} x\left(1+B^{2}\right)^{\frac{1}{2}},
$$

where $V_{\perp}$ is the volume in the perpendicular directions $y_{\perp}$ (we can think of the perpendicular directions as being compactified on a torus) and $T_{25}$ is the tension of the 25-brane. On the other hand, at the point $g=0$ in parameter space clearly all beta-functions $\beta^{i}$ vanish, and therefore $S(0)=Z(0)$. This implies that the normalization for the path-integral which defines $Z(g)$ for general values of the couplings is given by

$$
\langle\cdots\rangle_{I_{0}}=\int D X e^{-I_{0}(X)} \cdots \rightarrow T_{25} V_{\perp} \int d^{2} x\left(1+B^{2}\right)^{\frac{1}{2}} \int D \xi e^{-I_{0}(\xi)} \cdots,
$$

where the integral over the fields $X^{a}=x^{a}+\xi^{a}$ is given by an integral over the zero modes $x^{a}$ and an integral over the non-zero modes $\xi^{a}$, normalized so that $\int D \xi e^{-I_{0}(\xi)} \mathbf{1}=1$.

We now consider a generic tachyon profile given by an arbitrary function $t(x)$. The associated boundary operator is nothing but

$$
V=t(X)
$$


We will show that, in the limit of large magnetic field $B \rightarrow \infty$, we are able to compute both the partition function $Z(t)$, and the corresponding action $S(t)$. To this end, we first recall [1] that, for generic functions $f_{1}(x), \cdots, f_{n}(x)$, and for generic points $\tau_{1}, \cdots, \tau_{n}$ cyclically ordered on the boundary of the world-sheet $\Sigma$, one has that, in the limit $B \rightarrow \infty$,

$$
\left\langle f_{1}\left(X\left(\tau_{1}\right)\right) \cdots f_{n}\left(X\left(\tau_{n}\right)\right)\right\rangle_{I_{0}} \simeq T_{25} V_{\perp} \int d^{2} x B\left(f_{1} \star \cdots \star f_{n}\right) .
$$

In the above equation, the $\star$-product is given as usual [1] by $f \star g=f g+\frac{i}{2} \theta^{a b} \partial_{a} f \partial_{b} g+$ $\cdots$, with $\theta^{12}=\theta=B^{-1}$. Therefore, in the large $B$ limit, one can compute the partition function

$$
\begin{aligned}
Z(t) & =\left\langle\exp \left(-\frac{1}{2 \pi} \int t(X(\tau)) d \tau\right)\right\rangle_{I_{0}} \\
& \simeq V_{\perp} T_{25} \int d^{2} x B\left(1-t+\frac{1}{2} t \star t+\cdots\right) \\
& =V_{\perp} T_{25} \int d^{2} x B e^{-t} .
\end{aligned}
$$

We now rewrite the above result in terms of operators acting on a one-particle quantum Hilbert space. This is easily done using the standard function-operator correspondence (for example, see [24]), which assigns to each function $f(x)$ an operator $Q_{f}$ such that $Q_{f} \cdot Q_{g}=Q_{f \star g}$ and such that $\operatorname{Tr}\left(Q_{f}\right)=\frac{1}{2 \pi} \int d^{2} x B f$. We can then rewrite the partition function $Z$ as a function of the operator

$$
T=Q_{t}
$$

as

$$
Z(T)=2 \pi V_{\perp} T_{25} \operatorname{Tr}\left(e^{-T}\right) .
$$

We now compute the action $S$ as a function of the tachyon field. This is extremely simple in the limit of large $B$, since all the anomalous dimensions of boundary operators go to zero [1]. To quickly realize this fact, one must simply recall that the anomalous dimension of composite operators comes from the logarithmic divergence of the two-point function $\left\langle X^{a} X^{b}\right\rangle$ on the boundary of the string world-sheet $\Sigma$, which is proportional [1] to the so called open string metric $G^{a b} \simeq-\left(B^{-2}\right)^{a b}$. In the large $B$ limit, $G^{a b}$ vanishes, and so do all anomalous dimensions. This implies 
that the $\beta$-function associated to the tachyon field is simply given by

$$
\beta_{t(x)}=-t(x)
$$

Therefore, equation (2) implies that the action is given by

$$
\begin{aligned}
S(t) & =\left(1-\int d^{2} x t(x) \frac{\delta}{\delta t(x)}\right) Z(t) \\
& =V_{\perp} T_{25} \int d^{2} x B(1+t) e^{-t}
\end{aligned}
$$

or, in operator language, by

$$
S(T)=2 \pi V_{\perp} T_{25} \operatorname{Tr}\left[(1+T) e^{-T}\right] .
$$

This result could have been expected. One can in fact guess the above equation by considering the action proposed in [21, 22], and by replacing, as usual [1], ordinary products with $\star$-products. In the large $B$ limit, the derivative corrections become negligible, and one recovers (3). The point of this letter is to show that the formalism of background independent open string field theory actually confirms this expectation.

We now wish to describe solitons of the above theory which represent a single 23-brane. To this end, we follow the reasoning of [22] and consider only tachyon configurations which are quadratic in the coordinates $x^{a}$ (and also, given the symmetry of the problem, rotationally invariant)

$$
t(x)=c+u B r^{2}
$$

with $r^{2}=\left(x^{1}\right)^{2}+\left(x^{2}\right)^{2}$. Recalling the function-operator correspondence

$$
\frac{1}{\sqrt{2 \theta}}\left(x^{1} \pm i x^{2}\right) \rightarrow a, a^{\dagger}
$$

(where $\left[a, a^{\dagger}\right]=1$ ), we quickly see that the corresponding tachyon operator $T$ is given by

$$
T=c+u(2 N+1)
$$

\footnotetext{
${ }^{1}$ Also, no other field will be generated under RG flow, if it is not present from the start in the boundary action. It is therefore consistent to restric the string field space to only tachyon configurations.
} 
where $N=a^{\dagger} a$ is the number operator. Therefore, the partition function is given by

$$
\begin{aligned}
Z & =2 \pi V_{\perp} T_{25} e^{-c-u} \operatorname{Tr}\left(e^{-2 u N}\right) \\
& =2 \pi V_{\perp} T_{25} \frac{e^{-c-u}}{1-e^{-2 u}}
\end{aligned}
$$

Correspondingly, the action $S$ is

$$
\begin{aligned}
S & =\left(1-c \frac{\partial}{\partial c}-u \frac{\partial}{\partial u}\right) Z \\
& =2 \pi V_{\perp} T_{25} e^{-c} \frac{(u+1+c) e^{-u}+(u-1-c) e^{-3 u}}{\left(1-e^{-2 u}\right)^{2}}
\end{aligned}
$$

We now wish to find the stationary point of $S$ which corresponds to the 23-brane solution. This will surely correspond to a saddle point, since all branes are unstable to decay into the vacuum solution. In fact, we can first find a maximum of $S$ with respect to $c$ at the following value

$$
c^{\star}(u)=-u\left(\frac{1+e^{-2 u}}{1-e^{-2 u}}\right) .
$$

We can then follow the value of the action as a function of $c^{\star}, u$

$$
S\left(c^{\star}(u), u\right)=2 \pi V_{\perp} T_{25} \frac{1}{1-e^{-2 u}} \exp \left(\frac{2 u}{e^{2 u}-1}\right),
$$

which clearly decreases to the value

$$
S\left(c^{\star}(u), u\right) \rightarrow 2 \pi V_{\perp} T_{25}=T_{23} V_{\perp}
$$

at the saddle point obtained at $u \rightarrow \infty, c \rightarrow-\infty$, with $c \simeq-u$. This shows, like previously in [25, 22], that the energy of the soliton is exactly that of the 23-brane, as expected. Moreover the saddle point is at infinite values of the couplings $u$ and c. This is again to be expected (as also noted in [22) since infrared fixed points of $\mathrm{RG}$ flows always occur at infinite value of the couplings if one chooses coordinates in coupling space such that the $\beta$-functions are linear.

Some comments are in order:

1. First of all, the 23-brane soliton corresponds to the highly singular tachyon profile $t \simeq u\left(-1+B r^{2}\right)$ for $u \rightarrow \infty$. This might, at first sight, contradict the intuition [24, 25] that the solitons describing lower dimensional branes in the 
large magnetic field limit have a characteristic size of order $\sqrt{\theta}-$ i.e. $B r^{2} \sim 1$. In the limit $u \rightarrow \infty$, since the size of the soliton $B r^{2} \sim \frac{1}{u}$ is going to zero, one might worry that, even in the highly non-commutative limit, derivative corrections to the tachyon action are actually important in determining the energy of the solitonic configuration. This is not the case, as can be seen if one considers [22 the (highly non-local) field redefinition $\Phi=e^{-T / 2}$. In terms of this new variable the minimum of the tachyon potential is at $\Phi=0$ and the maximum at $\Phi=1$. Following the reasoning of [24, 25], we then expect the soliton to be simply given by $\Phi=\pi_{0}$, where $\pi_{n}=|n\rangle\langle n|$ is the projection onto the harmonic oscillator $n$-th state. This is indeed the case, since a tachyon configuration $t \simeq u\left(-1+B r^{2}\right)$ corresponds to an operator $T \simeq 2 u N$, or to

$$
\Phi \simeq e^{-u N} \rightarrow \pi_{0}
$$

for $u \rightarrow \infty$.

2. More generally, following the considerations of [24, 25], and using the intuition from the above remark, we see clearly that a solitons with $n$ coincident $23-$ branes can be described, within this framework, with a tachyon profile which is a polynomial of degree $2 n$ in the coordinate variables. Let us illustrate this fact in the case of $n=2$. It is simpler to proceed backwards, starting from the result of [24, 25]

$$
\Phi=\pi_{0}+\pi_{1}
$$

We can write the above equation as the limit, for $u \rightarrow \infty$, of

$$
\Phi=e^{-2 u N(N-1)} .
$$

Therefore we conclude that the correct tachyon configuration is given by the operator $T=4 u N(N-1)$ or, using $r^{2} \star r^{2}=r^{4}-\theta^{2}$, by the function

$$
t=u\left(B^{2} r^{4}-4 B r^{2}+1\right), \quad(u \rightarrow \infty)
$$

which is quartic in the coordinate variables, as claimed.

\section{Conclusions}

We have computed, using background independent open string field theory, both the tachyon potential, as well as the explicit form and the energies of soliton solutions 
corresponding to lower dimensional branes. This was achieved by considering a closed string background with large $B_{a b}$ field. In this case, the partition function which defines the theory is easily computed. Moreover, all anomalous dimensions vanish in the limit of large $B_{a b}$, and the $\beta$-function flow in the space of couplings, which is needed to compute the string action, is extremely simple, and can be analyzed explicitly for generic tachyon profiles.

One now has a handle, within background independent open string field theory, on the theory in two different closed string backgrounds. On the other hand, it is conjectured [12, 13], that the end-points of tachyon condensations actually correspond to the same point in configuration space of string field theory. In one description $\left(B_{a b}=0\right)$ all derivative $\alpha^{\prime}$ corrections count, but in the other $\left(B_{a b} \rightarrow \infty\right)$ none of them do. How this independence on the background $B_{a b}$ is achieved at the level of string field actions is still unclear, and is an open problem for future research.

\section{Acknowledgments}

This research is supported by a European Post-doctoral Institute Fellowship.

\section{References}

[1] N. Seiberg and E. Witten, "String theory and noncommutative geometry", JHEP 9909, 032 (1999), hep-th/9908142.

[2] V. A. Kostelecky and S. Samuel, "The static tachyon potential in the open bosonic string theory," Phys. Lett. B 207 (1988) 169.

[3] A. Sen and B. Zwiebach, "On a nonperturbative vacuum for the open bosonic string," Nucl. Phys. B 336 (1990) 263.

[4] A. Sen and B. Zwiebach, "Tachyon condensation in string field theory," hep-th/9912249, JHEP 0003 (2000) 002.

[5] N. Moeller and W. Taylor, "Level truncation and the tachyon in open bosonic string field theory," hep-th/0002237, Nucl. Phys. B 583 (2000) 105.

[6] V. A. Kostelecky and R. Potting, "Analytical construction of a nonperturbative vacuum for the open bosonic string", hep-th/0008252.

[7] J. A. Harvey and P. Kraus, "D-branes as unstable lumps in bosonic open string theory," hep-th/0002117, JHEP 0004 (2000) 012. 
[8] R. de Mello Koch, A. Jevicki, M. Mihailescu, and R. Tatar, "Lumps and pbranes in open string field theory," hep-th/0003031, Phys. Lett. B 482 (2000) 249 .

[9] N. Moeller, A. Sen and B. Zwiebach, "D-branes as tachyon lumps in string field theory," hep-th/0005036, JHEP 0008 (2000) 039.

[10] E. Witten, "Noncommutative geometry and string field theory," Nucl. Phys. B 268 (1986) 253.

[11] D. Ghoshal and A. Sen, "Normalisation of the background independent open string field theory action," hep-th/0009191.

[12] A. Sen, "Uniqueness of tachyonic solitons," hep-th/0009090.

[13] A. Sen, "Some issues in non-commutative tachyon condensation," hep-th/0009038.

[14] A. Sen, "Non-BPS states and branes in string theory," hep-th/9904207.

[15] A. Sen, "Tachyon condensation on the brane antibrane system," JHEP 9808, 012 (1998).

[16] A. S. Fradkin and A. A. Tseytlin, "Quantum String Theory Effective Action", Nucl. Phys. B261, 1-27 (1985).

[17] E. Witten, "On background independent open string field theory," Phys. Rev. D46, 5467 (1992).

[18] E. Witten, "Some computations in background independent off-shell string theory," Phys. Rev. D47, 3405 (1993).

[19] S. L. Shatashvili, "Comment on the background independent open string theory," Phys. Lett. B311, 83 (1993).

[20] S. L. Shatashvili, "On the problems with background independence in string theory," hep-th/9311177.

[21] A. Gerasimov and S. Shatashvili, "On exact tachyon potential in open string field theory," hep-th/0009103.

[22] D. Kutasov, M. Mariño and G. Moore, "Some exact results on tachyon condensation in string field theory", hep-th/0009148. 
[23] R. Gopakumar, S. Minwalla and A. Strominger, "Symmetry restoration and tachyon condensation in open string theory," hep-th/0007226.

[24] R. Gopakumar, S. Minwalla and A. Strominger, "Noncommutative solitons," JHEP 0005, 020 (2000).

[25] A. Harvey, P. Kraus, F. Larsen and E. J. Martinec, "D-branes and strings as non-commutative solitons," hep-th/0005031. 